Funding Open Access funding provided by Projekt DEAL.

Open Access Dieser Artikel wird unter der Creative Commons Namensnennung 4.0 International Lizenz veröffentlicht, welche die Nutzung, Vervielfältigung, Bearbeitung, Verbreitung und Wiedergabe in jeglichem Medium und Format erlaubt, sofern Sie den/die ursprünglichen Autor(en) und die Quelle ordnungsgemäß nennen, einen Link zur Creative Commons Lizenz beifügen und angeben, ob Änderungen vorgenommen wurden.

Die in diesem Artikel enthaltenen Bilder und sonstiges Drittmaterial unterliegen ebenfalls der genannten Creative Commons Lizenz, sofern sich aus der Abbildungslegende nichts anderes ergibt. Sofern das betreffende Material nicht unter der genannten Creative Commons Lizenz steht und die betreffende Handlung nicht nach gesetzlichen Vorschriften erlaubt ist, ist für die oben aufgeführten Weiterverwendungen des Materials die Einwilligung des jeweiligen Rechteinhabers einzuholen.

Weitere Details zur Lizenz entnehmen Sie bitte der Lizenzinformation auf http://creativecommons.org/ licenses/by/4.0/deed.de.

\title{
Bungert, Heike/Weiß, Jana (Hrsg.): ,God Bless America“. Zivilreligion in den USA im 20. Jahrhundert, 387 S., Campus, Frankfurt a. M./New York 2017.
}

\section{Andreas Linsenmann}

Online publiziert: 19. Mai 2020

(C) Der/die Autor(en) 2020

Der Orientierungsbedarf im Hinblick auf Strukturmerkmale und Dynamiken der politischen Kultur der USA ist, obschon ohnehin konstant hoch, in den vergangenen Jahren nochmals gestiegen - getrieben nicht zuletzt durch das Bedürfnis, die Wahl Donald Trumps zum US-Präsidenten sowie deren Auswirkungen einordnen und verstehen zu können. Der vorliegende Sammelband, hervorgegangen aus einem Workshop des Centrums für Religion und Moderne und des Exzellenzclusters „Religion und Politik“ der Westfälischen Wilhelms-Universität Münster, bietet hierzu instruktive Ansätze. Er reiht sich ein in eine Neubefragung des für die dominanten Identitätskonstruktionen der USA wichtigen und seit den Anschlägen vom 11. September 2001 merklich revitalisierten Konzepts der Zivilreligion (civil religion). Dieser auf Jean-Jacques Rousseaus Hauptwerk „Le Contrat Social“ (1762) zurückgehende Ter-

\footnotetext{
A. Linsenmann $(\bowtie)$

Universität Koblenz-Landau, Koblenz, Deutschland

E-Mail: linsenmann@uni-koblenz.de
} 
minus bezeichnet einen komplexen Apparat von Ideologien, Geschichtsdeutungen und Praktiken, die zu einer emotionalen Loyalität und quasireligiösen Verehrung beitragen, die US-Bürger in einem hohen Maß kollektiver Übereinstimmung ihrem Staat entgegenbringen. Ausgehend von dem Postulat, dass es zur Zivilreligion einen Überhang theoretischer Beiträge und ein Defizit empirischer Forschung gebe, bietet der Band nach einer das Terrain konzise definitorisch umreißenden Einleitung der Herausgeber zehn Beiträge von (Religions-)Historikern, Politologen, Kommunikationswissenschaftlern und Amerikanisten.

In einer ersten Sektion zur zivilreligiösen Aufladung von Ritualen wie Jubiläen, Wahlkämpfen und Sportveranstaltungen untersucht Heike Bungert Erinnerungsfeiern zu exponierten Daten der Besiedelung Nordamerikas, wie etwa die Gründung von Jamestown (1607), auf die In- und Exklusion von Gruppen sowie politische Codierungen. Dabei zeigt sie, wie kontextabhängig die jeweilige Ausgestaltung war. David Weiss analysiert, die lange Tradition der Presidential Rhetoric Studies aufgreifend, die Rhetorik George W. Bushs und präpariert eine ,,persönliche politischreligiöse Rhetorik“ heraus, mit der der 41. US-Präsident dem Religiösen einen Vorrang gegenüber der staatlichen Sphäre zugebilligt habe. Antonio Dantoro nimmt das Verhältnis von Sport und Zivilreligion in den Blick und zeigt unter anderem zivilreligiöse Überhöhungen der National Football League auf.

In der zweiten Sektion „Kriegs- und Krisenzeiten“ legt Morten Brænder die Rolle zivilreligiöser Rituale in Kriegskonstellationen dar. Jonathan H. Ebel greift diesen Zugang auf und analysiert vertiefend das Verschmelzen von Amerikanismus, Christentum und Kriegserinnerung. Dabei stellt er mit einer anregenden Thesenbildung einen Antagonismus zwischen mit Christus identifizierten „,durchschnittlichen“ Opfern und im Dissens zur Kriegspolitik befindlichen individuellen „Judas-Figuren“ zur Diskussion. Jana Weiß unterzieht Feiern des Independence Day sowie des Memorial Day vor und während des Vietnamkriegs einem close reading, wobei sie auch zivilreligiöse Bezugnahmen von Veteranen aufzeigt und damit ganz grundsätzlich Aneignungskonflikte sowie Fragen der Deutungshoheit ins Sichtfeld rückt. Raymond J. Haberski Jr. schließlich arbeitet sowohl Zustimmung als auch Kritik zu zivilreligiösen Konstellationen bei prominenten religiösen Intellektuellen heraus.

Die dritte Sektion öffnet das Feld der religiösen Gruppen und ethnischen Minderheiten. Anja-Maria Bassimir zeigt auf, dass Evangelikale Phänomenen der Zivilreligion lange als potenzieller Häresie mit Argwohn begegneten. Ulrike Stockhausen fasst den Gegenstand noch enger, indem sie zivilreligiöse Argumentationsstrategien bei evangelikalen Latinos in der Debatte um eine Einwanderungsreform untersucht. Andrew Manis sichtet abschließend in weit gefasster Perspektive bis zur Gegenwart Adaptions- und Abgrenzungsprozesse, die sich im Umgang von Afroamerikanern mit zivilreligiösen Kulturmustern feststellen lassen.

Der Band weist kleinere Schwächen auf. So wird der Begriff Zivilreligion teils unscharf verwendet und implizit mit dem Religiösen in der öffentlichen Sphäre gleichgesetzt. Letzteres deckt sich jedoch nicht zwingend mit dem Konzept Zivilreligion und blendet wichtige Dimensionen dieses Sets kohäsionsstiftender Narrative und Praktiken aus, deren verbindendes Merkmal insbesondere eine auf Affirmation zielende sakrale Imprägnierung von Staat, Nation und sozio-kulturellem Status quo darstellt. Ferner bleiben für die civil-religion-Debatte maßgebliche Positionen wie 
etwa Gunnar Myrdals einflussreiche Studie „An American Dilemma: The Negro Problem and Modern Democracy“ (1944), in der Myrdal ein gemeinschaftliches patriotisches und universell sendungsbewusstes American Creed als Grundlage für die Einheit der disparaten Nation USA beschrieb, weitgehend ausgeblendet. Auch scheint der Anspruch, mit zehn Themenbeiträgen einen Überblick über das Forschungsfeld zu bieten, zu weit gegriffen, zumal wichtige Disziplinen wie soziologisch geschärfte Perspektiven fehlen.

Diese Gravamina sollten jedoch die Stärken des Bandes keinesfalls überdecken. $\mathrm{Zu}$ diesen zählen, dass sämtliche Beiträge auf hohem Niveau rangieren, die empirischen Erträge beträchtlich sind und die Publikation insgesamt durchweg mit Gewinn zu lesen ist. Zweifellos bietet sie damit auch Orientierungs- und Erklärungsangebote für aktuelle Probleme und Fragestellungen.

Funding Open Access funding provided by Projekt DEAL.

Open Access Dieser Artikel wird unter der Creative Commons Namensnennung 4.0 International Lizenz veröffentlicht, welche die Nutzung, Vervielfältigung, Bearbeitung, Verbreitung und Wiedergabe in jeglichem Medium und Format erlaubt, sofern Sie den/die ursprünglichen Autor(en) und die Quelle ordnungsgemäß nennen, einen Link zur Creative Commons Lizenz beifügen und angeben, ob Änderungen vorgenommen wurden.

Die in diesem Artikel enthaltenen Bilder und sonstiges Drittmaterial unterliegen ebenfalls der genannten Creative Commons Lizenz, sofern sich aus der Abbildungslegende nichts anderes ergibt. Sofern das betreffende Material nicht unter der genannten Creative Commons Lizenz steht und die betreffende Handlung nicht nach gesetzlichen Vorschriften erlaubt ist, ist für die oben aufgeführten Weiterverwendungen des Materials die Einwilligung des jeweiligen Rechteinhabers einzuholen.

Weitere Details zur Lizenz entnehmen Sie bitte der Lizenzinformation auf http://creativecommons.org/ licenses/by/4.0/deed.de. 